\title{
REPRESENTATION THEOREMS FOR ANALYTIC FUNCTIONS WITH QUASIMEROMORPHIC EXTENSIONS
}

\author{
Zerrin Göktürk
}

This paper is concerned with normalized quasimeromorphic functions of the extended plane $\overline{\mathbf{C}}$ which are analytic in a domain $D$ of the plane, and have a pole only at one point. For these functions, which are strictly finitely multivalent in $\overline{\mathbf{C}}$, we generalize representation theorems concerning normalized quasiconformal homeomorphisms of $\overline{\mathbf{C}}$ which are conformal in $D$. The representation formulas yield estimates for the power series coefficients.

\section{Definitions}

A function $f$ is called $k$-quasimeromorphic in a plane domain $D$, if $f$ is spherically continuous and a generalized $L^{2}$-solution of a Beltrami differential equation $f_{\bar{z}}=\mu f_{z}$ in $D$, where the complex dilatation $\mu$ satisfies the condition $\|\mu\|_{\infty} \leq k<1$.

We introduce the class $F_{k}^{p}$ of $k$-quasimeromoprhic functions $f$ of $\overline{\mathbf{C}}$ whose restrictions to $D^{*}=\{z|| z \mid>1\}$ are meromorphic and of the form

$$
f(z)=\sum_{n=0}^{p} a_{n} z^{p-n}+\sum_{n=1}^{\infty} a_{p+n} z^{-n}, \quad a_{0}=1, p \in N
$$

and $f(z)=\infty$ only for $z=\infty$.

$\sum_{k}^{p}$ denotes the subclass of $F_{k}^{p}$ consisting of functions $f$ whose singular part at $\infty$ reduces to $z^{p}[2]$ :

We denote by $\sum_{k}$ the class of $k$-quasiconformal homeomorphisms $f$ of $\overline{\mathbf{C}}$ which are conformal in $D^{*}$ with a development of the form

$$
f(z)=z+\sum_{n=1}^{\infty} b_{n} z^{-n}
$$

The research was supported by the Academy of Finland. 


\section{Representation theorems}

Theorem 2.1. A function $f \in F_{k}^{p}$ has the representation (2.1)

$$
f=P \circ h
$$

where $h \in \sum_{k}$ and $P$ is a polynomial of degree $p$ with leading coefficient 1 .

Proof. Let $f \in F_{k}^{p}$ and $\mu$ be its complex dilatation. From the existence and uniqueness theorems for the Beltrami equation it follows that there is a unique quasiconformal homeomorphism $h \in \sum_{k}$ with complex dilatation $\mu_{h}=\mu$ a.e. The function $P=f \circ h^{-1}$ has then the complex dilatation zero a.e. in C. Since it has $L^{1}$-derivatives, it is meromorphic in $\overline{\mathbf{C}}$, and hence rational. Because $f$ has the only pole at $z=\infty, P$ is a polynomial. If follows from the normalization (1.1) that it is a polynomial of degree $p$ with leading coefficient 1.

Let $f \in \sum_{k}^{p}$. Then the polynomial $P$ in (2.1) is the Faber polynomial of degree $p$ of $h$, since the only polynomial of degree $p$ such that the singular part of $P[h(z)]$ at $\infty$ reduces to $z^{p}$ is the $p^{\text {th }}$ Faber polynomial of $h$.

Remark 2.1. Let $L_{0, k}^{\infty}$ denote the set of complex valued measurable functions $\mu$ satisfying $\|\mu\|_{\infty} \leq k<1$ and having support in the closure of the unit disc $D$. A function $\mu \in L_{0, k}^{\infty}$ determines uniquely the element $h \in \sum_{k}$ whose complex dilatation $\mu_{h}$ equals $\mu$ a.e. but not the element of $F_{k}^{p}$. For, if $P$ is an arbitrary polynomial of degree $p$ with leading coefficient 1 , then $f=P \circ h \in F_{k}^{p}$ with $\mu_{h}=\mu$ a.e. However there is a one-to-one correspondence between the functions $\mu \in L_{0, k}^{\infty}$ and the elements $f \in \sum_{k}^{p}$. In this case the uniqueness (and the existence) of $f \in \sum_{k}^{p}$ follows from the uniqueness (the existence) of the Faber polynomials.

It is an immediate consequence of Theorem 2.1 that a function $f \in F_{k}^{p}$ takes each value in $\overline{\mathbf{C}}$ exactly $p$ times. In particular, a function $f \in F_{k}^{1}$ is a homeomorphism, a translation of its basic homeomorphism $h \in \sum_{k}$. Moreover, since 0 -quasimeromorphic functions are meromorphic, $F_{0}^{p}$ is the set of all polynomials of degree $p$ with leading coefficient 1 . We use the same notation $F_{k}^{p}$ for the class of the restrictions $\left.f\right|_{D^{*}}$ of all $f \in F_{k}^{p}$. Then every $F_{k}^{p}, 0 \leq k<1$, is contained in the class $F^{p}$ of functions $f$ which take every value at most $p$ times in $D^{*}$ and have a development of the form (1.1).

A function $g$ which is analytic in the interior of $C_{R}=h(|z|=R)$ for some $R \in(1, \infty)$ can be expanded into a series of Faber polynomials belonging to $h$, i.e., the function $g$ has the representation

$$
g(w)=\sum_{m=0}^{\infty} c_{m} P_{m}(w)
$$

in the interior of $C_{R}$, where $P_{m}$ denotes the $m^{\text {th }}$ Faber polynomial of $h$, and

$$
c_{m}=\frac{1}{2 \pi} \int_{|z|=\varrho} g(h(z)) z^{-m-1} d z, \quad \varrho \in(1, R), m=0,1, \ldots
$$

The representation is unique [8], [11]. 
Theorem 2.2. Let $f \in F_{k}^{p}$. Then $f$ has the representation

$$
f=f_{p}+a_{1} f_{p-1}+\cdots+a_{p-1} f_{1}+a_{p},
$$

where $f_{m}=P_{m} \circ h \in \sum_{k}^{m}, m=1, \ldots, p$, and $a_{m}$ are the power series coefficients of $f$.

Proof. Let $f \in F_{k}^{p}$. By Theorem $2.1 f$ has a representation of the form (2.1). Expanding $P$ into series in terms of Faber polynomials $P_{m}$ of $h$ we obtain

$$
P(w)=\sum_{m=0}^{p} c_{m} P_{m}(w)
$$

where the $c_{m}$ are given by (2.2). It is clear that $c_{m}=a_{p-m}, m=0, \ldots, p$. Hence, $f=P \circ h=\left(\sum c_{m} P_{m}\right) \circ h$ and the assertion follows.

Consequently, for a given polynomial $G(z)=\sum_{n=0}^{p} a_{n} z^{p-n}, a_{0}=1$, and a function $\mu \in L_{0, k}^{\infty}$ there exists a unique quasimeromorphic function $f \in F_{k}^{p}$ which has complex dilatation $\mu_{f}=\mu$ a.e., and $G$ as its principal part at $z=\infty$.

Let $T$ be the operator defined by

$$
T w(z)=-\frac{1}{\pi} \iint_{\mathbf{C}} \frac{w(\zeta)}{\zeta-z} d \xi d \eta, \quad \zeta=\xi+i \eta
$$

and $H$ the two-dimensional Hilbert transformation ([9] Chapter III.7). It is well known that using $T$ and $H$, a function of $\sum_{k}$ can be represented with the aid of its complex dilatation. The proof of the representation formula ([9], Chapter V.5) applies, with obvious modifications, to the functions of $F_{k}^{p}$ also.

Theorem 2.3. Let $f \in F_{k}^{p}$. Then

$$
f(z)=G(z)+\sum_{n=1}^{\infty} T \phi_{n}(z), \quad z \in \mathbf{C},
$$

where $G$ is the principal part of $f$ at $z=\infty$ and the functions $\phi_{n}$ are defined by $\phi_{1}=\mu G^{\prime}, \phi_{n}=\mu H \phi_{n-1}, n=2,3, \ldots$ The series is uniformly convergent .

Just as in the case $p=1$, formula (2.3) gives asymptotic estimates for the coefficients of the functions in $F_{k}^{p}$.

Theorem 2.4. Let $f$ belong to $F_{k}^{p}$ and have the expansion (1.1). Then

$$
\left|a_{p+n}\right| \leq 2 k \sum_{m=0}^{p-1} \frac{p-m}{p+n-m}\left|a_{m}\right|+O\left(k^{2}\right), \quad n=1,2, \ldots
$$

The estimate is sharp. 
The proof given in [10], pp. 432-433, for the counterpart of (2.4) in $\sum_{k}$ can be repeated as such, with the only difference that now $\phi_{1}=\mu G^{\prime}$.

Equality holds for the functions

$$
f_{n}=f_{n, p}+a_{1} f_{n,(p-1)}+\cdots+a_{p-1} f_{n, 1}+a_{p}
$$

where the functions $f_{n, m}$ are defined by

$$
f_{n, m}(z)= \begin{cases}\left(z^{(m+n) / 2}+k z^{-(m+n) / 2}\right)^{2 m /(m+n)} & \text { for }|z|>1, \\ \left(z^{(m+n) / 2}+k \bar{z}^{(m+n) / 2}\right)^{2 m /(m+n)} & \text { for }|z| \leq 1,\end{cases}
$$

for $m=1, \ldots, p$.

For the special case $G(z)=z^{p}$, inequality (2.4) yields the sharp estimate

$$
\left|a_{p+n}\right| \leq \frac{2 p k}{p+n}+O\left(k^{2}\right), \quad n=1,2, \ldots
$$

Let $F_{k}^{p}(\zeta)$ denote the class of functions $f$ in $F_{k}^{p}$ which take the value zero only at the point $\zeta$. If $f \in F_{k}^{p}(\zeta)$, then it follows from Theorem 2.1 that

$$
f=(h-h(\zeta))^{p}
$$

where $h \in \sum_{k}$.

Theorem 2.5. Let $f \in F_{k}^{p}(0)$. Then

$$
\left|a_{1}\right| \leq 2 p k \text {. }
$$

Equality holds only for the functions

$$
f(z)= \begin{cases}\left(z^{1 / 2}+k e^{i \theta} z^{-1 / 2}\right)^{2 p} & \text { for }|z|>1 \\ \left(z^{1 / 2}+k e^{i \theta} \bar{z}^{1 / 2}\right)^{2 p} & \text { for }|z| \leq 1\end{cases}
$$

Proof. The estimate (2.6) follows from $f=(h-h(0))^{p}$ when we take into account Kühnau's result $|h(0)| \leq 2 k$ ([5]).

In Section 3 we shall derive the above estimates from a general inequality.

Let $f=(h-h(\zeta))^{p} \in F_{k}^{p}(\zeta)$ and let $b_{n}, n=1,2, \ldots$, denote the power series coefficients of $h$. We see that $a_{1}=a_{2}=\cdots=a_{N}=0$ if and only if $h(\zeta)=0$ and

$$
b_{1}=b_{2}=\cdots=b_{N-1}=0 .
$$

In this case,

$$
a_{n}=p b_{n-1}, \quad n=N+1, \ldots, 2 N+1 .
$$


Theorem 2.6. Let $f \in F_{k}^{p}(0)$. If $a_{n}=0, n=1,2, \ldots, N(N \geq 1)$, then

$$
\left|a_{n}\right| \leq \frac{2 k p}{n}, \quad n=N+1, \ldots, 2 N+1 .
$$

Equality holds for the functions

$$
f(z)= \begin{cases}\left(z^{n / 2}+k e^{i \theta} z^{-n / 2}\right)^{2 p / n} & \text { for }|z|>1, \\ \left(z^{n / 2}+k e^{i \theta} \bar{z}^{n / 2}\right)^{2 p / n} & \text { for }|z| \leq 1\end{cases}
$$

Proof. Again, we make use of (2.5). Because (2.7) is true, $\left|b_{n}\right| \leq 2 k /(n+1)$, $n=N, N+1, \ldots, 2 N$ (Kühnau [6]). Hence, (2.9) follows from (2.8).

In particular, for $N=1$ we have $\left|a_{2}\right| \leq k p$ and $\left|a_{3}\right| \leq 2 k p / 3$.

In [3] we proved that $\left|a_{1}\right| \leq 4 k / 3$ in $\sum_{k}^{2}$, which can also be deduced from (2.9). In $\sum_{k}^{p}$, the estimate $\left|a_{p}\right| \leq k$ holds true ([2]).

\section{Majorant principle for the class $F_{k}^{p}(\zeta)$}

In this section we establish a counterpart of Lehto's majorant principle [10] for the class $F_{k}^{p}(\zeta), \zeta \in \bar{D}$, from which we obtain estimates for the power series coefficients $a_{n}$ of a function $f \in F_{k}^{p}(\zeta)$. The estimate for $\left|a_{1}\right|$ leads to a distortion theorem for $|h|, h \in \sum_{k}$ in $\bar{D}$.

We denote by $F^{p}(0)$ the class of functions $f$ in $F^{p}$ which do not assume the value zero in $D^{*}$. Then every restricted class $F_{k}^{p}(\zeta), \zeta \in \bar{D}$, is contained in the class $F^{p}(0)$.

The classes $\sum_{k}$ and $\sum_{k}^{\prime}=\left\{\left.h\right|_{D^{*}} \mid h \in \sum_{k}\right\}, 0 \leq k<1$, are known to be compact in the topology of locally uniform convergence. From the representation (2.5) it follows that every $F_{k}^{p}(\zeta), 0 \leq k<1$, is compact. Also, $F^{p}(0)$ is compact.

Let $\Phi$ be an analytic functional defined on $F^{p}(0)$. Then $\Phi$ is defined on every $F_{k}^{p}(\zeta), 0 \leq k<1$. Because the classes $F^{p}(0)$ and $F_{k}^{p}(\zeta)$ are compact,

$$
\max _{f \in F^{p}(0)}|\Phi(f)|=M(1) \quad \text { and } \quad \max _{f \in F_{k}^{p}(\zeta)}|\Phi(f)|=M(k)
$$

exist. The class $F_{0}^{p}(\zeta)$ contains only the function $f_{0}=(z-\zeta)^{p}$, and we write $M(0)=\left|\Phi\left(f_{0}\right)\right|$.

Theorem 3.1. Let $\Phi$ be an analytic functional defined on $F^{p}(0)$. Then for every $f \in F_{k}^{p}(\zeta)$,

$$
M(1) \frac{M(0)-k M(1)}{M(1)-k M(0)} \leq|\Phi(f)| \leq M(1) \frac{M(0)+k M(1)}{M(1)+k M(0)} .
$$

In particular, if $\Phi\left(f_{0}\right)=0$,

$$
|\Phi(f)| \leq k M(1) .
$$

Proof. In [10], inequality (3.1) was established in the case $f \in \sum$. Thanks to the simple relation $(2.5)$ the same proof applies to $F_{k}^{p}(\zeta)$. 
Corollary 3.1. Let $f \in F_{k}^{p}(\zeta)$. Then

$$
\left|a_{1}\right| \leq 2 p \frac{|\zeta|+2 k}{2+k|\zeta|}
$$

Proof. Let $\Phi(f)=a_{1}$. Then $M(0)=p|\zeta|$ and by Theorem XI.6.3. in [1] we have $M(1)=2 p$. Thus (3.2) follows from the right-hand inequality of (3.1).

For the class $F_{k}^{1}(\zeta)$ we obtain from (3.1)

$$
\left|a_{1}\right| \leq 2 \frac{|\zeta|+2 k}{2+k|\zeta|}
$$

Corollary 3.2. Let $h \in \sum_{k}$. Then for $\zeta \in \bar{D}$

$$
|h(\zeta)| \leq 2 \frac{|\zeta|+2 k}{2+k|\zeta|}
$$

Proof. The function $f=(h-h(\zeta))^{p}$ is in $F_{k}^{p}(\zeta)$. Since $a_{1}=-p h(\zeta)$, the assertion follows from (3.2). As $k \rightarrow 1$, it gives the well-known sharp estimate $|h(\zeta)| \leq 2$ in $\sum$.

For $\zeta=0$ Corollary 3.2. yields the sharp estimate $|h(0)| \leq 2 k[5]$.

Corollary 3.3. Let $f \in F_{k}^{p}(0)$. Then

$$
\left|a_{1}\right| \leq 2 p k, \quad\left|a_{2}\right| \leq p(2 p-1) k .
$$

The first estimate is sharp.

Proof. The first estimate follows from Corollary 3.1 for $\zeta=0$.

For the second estimate, let $\Phi(f)=a_{2}$. For $\zeta=0, f_{0}(z)=z^{p}$ and therefore $M(0)=0$. By Theorem XI.6.3 in [1], $M(1)=p(2 p-1)$ and the assertion follows from (3.1).

For $p=1$ we obtain $\left|a_{1}\right| \leq 2 k,\left|a_{2}\right| \leq k$ for the class $F_{k}^{1}(0)[5,6]$.

Remark 3.1. The second estimate in Corollary 3.3. is not sharp for $p>1$. For, let $f \in F_{k}^{p}(0)$. By the representation (2.5)

$$
(f(z))^{1 / p}=h(z)-h(0)=z+\frac{a_{1}}{p}+\sum_{1}^{\infty} c_{n} z^{-n}
$$

and

$$
c_{1}=\frac{a_{2}}{p}-\frac{p-1}{2 p^{2}} a_{1}^{2} .
$$

Since $\left|c_{1}\right| \leq k$, this together with the first estimate in Corollary 3.3 yields

$$
\left|a_{2}\right| \leq p k+2 p(p-1) k^{2} .
$$

Remark 3.2. Let $\Phi(f)=a_{n}, n=3,4, \ldots$, for $f \in F^{p}(0)$. Then $M(0)=$ 0 and by Theorem XI.6.3 in [1], $M(1) \leq C(n, p)$, where $C(n, p)$ is a constant depending on $n$ and $p$ only. By Theorem 3.1 we have then $\left|a_{n}\right| \leq k C(n, p)$ in $F_{k}^{p}(0)$. We note that bounds of this kind cannot be always found for the class $F_{k}^{p}$. 


\section{Class $S_{k}^{p}(\zeta)$}

We denote by $S_{k}^{p}(\zeta)$ the class of $k$-quasimeromorphic functions $f$ of $\overline{\mathbf{C}}$ which are analytic in $D$ and of the form

$$
f(z)=z^{p}+\sum_{n=1}^{\infty} a_{p+n} z^{n}
$$

and $f(z)=0, \infty$ only for $z=0, \zeta$, respectively.

$S_{k}(\infty)$ denotes the class of $k$-quasiconformal homeomorphisms $f$ of $\overline{\mathbf{C}}$ whose restrictions to $D$ have the form

$$
f(z)=z+\sum_{n=2}^{\infty} b_{n} z^{n}
$$

and which leave $\infty$ fixed.

The proof of the following representation theorem is similar to the proof of Theorem 2.1.

Theorem 4.1. A function $f \in S_{k}^{p}(\zeta)$ has the unique representation

$$
f=\left(\frac{h}{1-h / h(\zeta)}\right)^{p}
$$

where $h \in S_{k}(\infty)$.

Consequently, a function $f \in S_{k}^{p}(\zeta)$ takes every value in $\overline{\mathbf{C}}$ exactly $p$ times. In particular, a function $f \in S_{k}^{1}(\zeta)$ is a homeomorphism. We write $S_{k}^{1}(\zeta)=S_{k}(\zeta)$.

Theorem 4.2. A function $f$ of $S_{k}^{p}(\zeta)$ has the unique representation

$$
f=(\tilde{h})^{p}
$$

where $\tilde{h} \in S_{k}(\zeta)$.

If $f \in S_{k}^{p}(\infty)$, the representation (4.2) takes the form

$$
f=h^{p}
$$

where $h \in S_{k}(\infty)([2])$.

It follows from Theorem 4.1 that the class $S_{0}^{p}(\zeta)$ contains only the function $f_{0}(z)=z^{p}(1-z / \zeta)^{-p}$. We use the same notation $S_{k}^{p}(\zeta)$ for the class of the restrictions $\left.f\right|_{D}$ of all $f \in S_{k}^{p}(\zeta)$. Then every $S_{k}^{p}(\zeta), 0 \leq k<1$, is contained in the class $S^{p}$ of analytic functions $f$ which take every value at most $p$ times in $D$ and have the normalization (4.1).

Majorant principle. Let $\Phi$ be an analytic functional defined on $S^{p}$. Again, the classes $S^{p}$ and $S_{k}^{p}(\zeta)$ are compact so that

$$
\max _{f \in S^{p}}|\Phi(f)|=M(1) \quad \text { and } \quad \max _{f \in S_{k}^{p}(\zeta)}|\Phi(f)|=M(k)
$$

exist. For the function $f_{0}=z^{p}(1-z / \zeta)^{-p}$ we write $M(0)=\left|\Phi\left(f_{0}\right)\right|$.

Theorem 3.1 applies for the classes $S_{k}^{p}(\zeta)$ and $S^{p}$ : If $\Phi$ is an analytic functional defined on $S^{p}$, then (3.1) holds for every $f \in S_{k}^{p}(\zeta)$. 
Corollary 4.1. Let $f \in S_{k}^{p}(\zeta)$. Then

$$
\left|a_{p+1}\right| \leq 2 p \frac{1+2 k|\zeta|}{2|\zeta|+k}
$$

Proof. Consider the analytic functional $\Phi(f)=a_{p+1}$. Then $M(1)=2 p$ by Theorem XI.6.5 in [1] and $M(0)=p /|\zeta|$. Thus the assertion follows from (3.1).

Corollary 4.2. Let $f \in S_{k}^{p}(\zeta)$. Then

$$
\left|a_{p+2}\right| \leq p(2 p+1) \frac{(p+1)+2 k(2 p+1)|\zeta|^{2}}{2(2 p+1)|\zeta|^{2}+k(p+1)} .
$$

Proof. Let $\Phi(f)=a_{p+2}$. Then $M(0)=p(p+1) / 2|\zeta|^{2}$, and by Corollary 8.16 in [4] $M(1)=p(2 p+1)$. Thus the assertion follows from (3.1).

We obtain from (4.4) and (4.5) the estimates

$$
\left|a_{2}\right| \leq \frac{1+2 k|\zeta|}{2|\zeta|+k}, \quad\left|a_{3}\right| \leq 3 \frac{1+3 k|\zeta|^{2}}{3|\zeta|^{2}+k}
$$

for the class $S_{k}(\zeta)$. Furthermore, $\left|a_{p+1}\right| \leq 2 p k^{1}$ and $\left|a_{p+2}\right| \leq p(2 p+1) k$ in $S_{k}^{p}(\infty)$. The first estimate is sharp.

Theorem 4.3. Let $f \in S_{k}^{p}(\infty)$. If $a_{p+n}=0, n=1, \ldots, N,(N \geq 1)$, then

$$
\left|a_{p+n}\right| \leq \frac{2 p k}{n}, \quad n=N+1, \ldots, 2 N+1 .
$$

Equality holds for the functions

$$
f(z)= \begin{cases}z^{p}\left(1+k e^{i \theta} z^{n}\right)^{-2 p / n} & \text { for }|z|<1 \\ (z \bar{z})^{p}\left(\bar{z}^{n / 2}+k e^{i \theta} z^{n / 2}\right)^{-2 p / n} & \text { for }|z| \geq 1\end{cases}
$$

Proof. The function $z \mapsto 1 / f(1 / z)$ is in $F_{k}^{p}(0)$. It has the expansion

$$
\frac{1}{f\left(\frac{1}{z}\right)}=z^{p}\left(1+\sum_{N+1}^{\infty} c_{n} z^{-n}\right)
$$

where $c_{n}=-a_{p+n}$ for $n=N+1, \ldots, 2 N+1$. Hence the assertion follows from Theorem 2.6.

1 A different proof of this estimate was given in [2]. 
Theorem 4.4. Let $f \in S_{k}^{p}(\infty)$. Then for $z \in \bar{D}$

$$
c^{p} \leq|f(z)| \leq C^{p},
$$

where $c$ and $C$ are Kühnau's constants [7]. The estimate is sharp.

The theorem follows from the representation (4.3) and Kühnau's distortion theorem ([7]).

Remark 4.1. Let $S$ denote the class of conformal homeomorphisms $f$ of $D$ which are normalized by the conditions $f(0)=0, f^{\prime}(0)=1$. It is known that the classes $S_{k}^{\prime}(\infty)=\left\{\left.h\right|_{D} \mid h \in S_{k}(\infty)\right\}, 0 \leq k<1$, are dense in $S$ with respect to the topology of locally uniform convergence, as $k \rightarrow 1$. However, the classes $S_{k}^{p}(\infty)$ are not dense in $S^{p}$, nor are $\sum_{k}^{p}$ dense in $\sum^{p}$. 


\section{Zerrin Göktürk}

\section{References}

[1] Goluzin, G.M.: Geometric theory of functions of of a complex variable. - American Mathematical Society Translations of Mathematical Monographs 26, Providence, R.I., 1969.

[2] Göктürk, Z.: On $p$-valent quasimeromorphic functions. - Proceedings of the Rolf Nevanlinna Symposium on Complex Analysis, Silivri 1976. Publications of the Nazım Terzioğlu Mathematical Research Institute 7, Istanbul, 1978.

[3] GökтÜRK, Z.: Remarks on $p$-valent quasimeromorphic functions. - Proceedings of the Vth Romanian-Finnish Seminar on Complex Analysis, Bucharest, 1981. Lecture Notes in Mathematics 1013. Springer-Verlag, Berlin-Heidelberg-New York, 1983.

[4] Jenkins, J.A.: Univalent functions and conformal mappings. - Ergebnisse der Mathematik 18, Springer-Verlag, Berlin, 1958.

[5] KüHNAU, R.: Wertannahmeprobleme bei quasikonformen Abbildungen mit ortsabhängiger Dilatationsbeschränkung. - Math. Nachr. 40, 1969, 1-11.

[6] KüHNAU, R.: Verzerrungsätze und Koeffizientenbedingungen von Grunskyschen Typ für quasikonforme Abbildungen. - Math. Nachr. 48, 1971, 77-105.

[7] KühnaU, R.: Eine Verschärfung des Koebeschen Viertelsatzes für quasikonform fortsetzbare Abbildungen. - Ann. Acad. Sci. Fenn. Ser. A I Math. 1, 1975, 77-83.

[8] Lebedev, N.A., and V.I. Smirnov: Functions of a complex variable. - London Iliffe Book Ltd., London, 1968.

[9] Lehto, O., and K.I. Virtanen: Quasiconformal mappings in the plane. - Grundlehren der mathematischen Wissenschaften 126. Springer-Verlag, New York-HeidelbergBerlin, 1973.

[10] Leнto, O.: Quasiconformal mappings and singular integrals. - Istituto Nazionale di Alta Matematica. Symposia Mathematica XVIII. Academic Press, Bologna, 1976.

[11] Schober, G.: Univalent functions. - Lecture Notes in Mathematics 478. Springer-Verlag, New York-Heidelberg-Berlin, 1975.

Boğaziçi University

Department of Mathematics

Istanbul

Turkey

Received 16 March 1984

Revised 31 May 1988 